ERRATUM

\title{
Comparison of a soluble co-formulation of insulin degludec/insulin aspart vs biphasic insulin aspart 30 in type 2 diabetes: a randomised trial
}

Leo Niskanen, Lawrence A Leiter, Edward Franek, Jianping Weng, Taner Damci, Manuel Muñoz-Torres, Jean-Paul Donnet, Lars Endahl, Trine Vang Skjøth and Allan Vaag

The journal and the authors apologise for errors in Table 2 of this article that was published in the August issue (vol 167, pp 287-294). The $n$ values were incorrectly published. The correct values are presented below and the table is published in full below.

European Journal of Endocrinology 167453

Table 2 Observed mean changes from baseline HbA1c, FPG and body weight. Data are observed as mean (s.D.) for all randomised subjects (full analysis set).

\begin{tabular}{llrrr}
\hline & $n$ & Baseline $^{\mathrm{a}}$ & Week 16 & Change from baseline \\
\hline HbA1c (\%) & & & & \\
IDegAsp & 61 & $8.5(1.2)$ & $6.7(1.0)$ & $-1.8(1.1)^{\mathrm{c}}$ \\
AF & 59 & $8.5(0.9)$ & $6.6(0.6)$ & $-1.9(1.1)^{\mathrm{c}}$ \\
BIAsp 30 & 62 & $8.6(1.0)$ & $6.7(0.7)$ & $-1.8(0.9)^{\mathrm{c}}$ \\
FPG (mmol/l) & & $11.5(2.6)$ & $6.4(2.2)$ & $-5.1(2.9)$ \\
IDegAsp & 61 & $11.8(2.9)$ & $6.5(1.9)$ & $-5.3(3.0)$ \\
AF & 59 & $11.7(3.1)$ & $7.5(2.1)$ & $-4.3(3.0)$ \\
BIAsp 30 & 62 & $87.5(16.3)$ & $88.6(16.9)$ & $1.1(2.8)$ \\
Body Weight (kg) & 61 & $84.9(14.3)$ & $85.6(14.9)$ & $0.7(2.5)$ \\
IDegAsp & 59 & $91.8(13.5)$ & $93.2(13.1)$ & $1.4(3.2)$ \\
AF & 62 & & \\
BIAsp 30 & &
\end{tabular}

${ }^{a}$ Values at randomisation.

bast observation carried forward.

${ }^{c} \%$ points. 